\section{Case 5}

A married woman aged 47 was seen at home on July 16 1952. Thirteen days previously she had a febrile illness diagnosed as influenza. Ten days later diffuse irritation occurred all over the body. The following day her eyes hurt on movement, and that night she had pain in the arms and legs-it felt as if it was " in the bones." The following morning she still had the pain, and found she could not read small print. During the course of the day the irritation of the skin increased. She felt " as if she had passed through fire." Trembling fits occurred which she could control voluntarily.

On the morning of July 16 she had diplopia, which had persisted. She had had retention of urine for seven hours, but later passed urine with difficulty. Her appetite was poor and she vomited once.

Clinically she complained of diplopia on looking to the left and blurring of vision on looking to the right, but there was no obvious ocular palsy or nystagmus; the optic fundi were normal, and the visual fields were not restricted. Her speech was normal, but her doctor and her husband commented that cerebration was slower. There was no loss of power in any limb, but the muscles were tender on pressure in the thighs, calves, and arms. All her deep reflexes were exceedingly brisk. The plantar reflexes were flexor. Apart from a doubtful loss to pin-prink sensation on the legs, there was no sensory change. The heart rate was 130 and regular, the heart sounds were normal, and the blood pressure was $135 / 95$. A blood count was normal, and also the urine.

She was admitted to hospital, where she was found to have a raised C.S.F. pressure, $90 \mathrm{mg}$. of proteins per $100 \mathrm{ml}$., and fewer than 5 cells per c.mm.

Two days after admission she developed generalized weakness of all limbs, together with poor diaphragm movements, and was transferred to the isolation hospital. Her deep reflexes became sluggish, but did not disappear, and at the same time she developed bilateral facial palsy. The respiratory paralysis did not develop, and she was transferred to Harefield Hospital. A further lumbar puncture on July 27 showed : protein, $80 \mathrm{mg}$. per $100 \mathrm{ml}$.; 1 lymphocyte per c.mm; chlorides, $750 \mathrm{mg}$. per $100 \mathrm{ml}$.; Lange 0000000000 . She improved rapidly, and was discharged on August 5, having completely recovered all facial and limb movements.

\section{Case 6}

A married woman aged 42 was admitted to the isolation hospital on July 30, 1953, with a history of pain behind the eyes and left facial palsy for two days. Six days previously she had had some pain in the back with general malaise. On the day before admission she developed a right facial palsy also. She had tingling in the hands and feet, but no other symptoms. On admission there was bilateral facial palsy, eye movements were full and normal, and muscle power was normal in all limbs. The ankle-jerks were absent, but knee-jerks were brisk. Within two days all her deep reflexes except the triceps-jerks disappeared. Lumbar puncture showed $110 \mathrm{mg}$. protein per $100 \mathrm{ml}$., and 1 polymorph cell only. Her blood count was normal.

She was transferred to Harefield Hospital on August 4. By this time, however, her deep reflexes had returned, though they were sluggish, but the ankle-jerks were still absent. Repeat lumbar puncture on August 17 showed $65 \mathrm{mg}$. of protein per $100 \mathrm{ml}$, and no cells. Recovery of facial movements was very slow, and she was discharged home on September 12 with a little but still incomplete movement of the face.

\section{Treatment}

No specific treatment was given other than physiotherapy when the acute stage had subsided.

\section{Summary}

Six cases of the Guillain-Barre syndrome are described. All had facial diplegia. In four of them, exaggeration of deep reflexes was observed in the pre-paralytic stage. Paraesthesiae and bilateral muscle tenderness followed by widespread paralysis of limbs were a feature in five of the cases.

I am grateful to Dr. J. T. Scott and Dr. A. Stuart for their help with these cases, and in particular to Dr. V. Lambert, of St. John's Isolation Hospital, for his willing help and co-operation at all times.

REFERENCES

Bannwarth, A. (1949), Lancet, 2, 1153

Bradford J. R., Bashford, E. F., and Wilson, J. A. (1919). Quart. J. Med., 12, 88

Guillain, G. (1936). Arch. Neurol. Psychiat., Chicago, 36, 975.

Barre, J. A., and Strohl, A. (1916). Bull. Soc. méd. Hop. Paris, 40, 1462 .

Holmes, G. (1917). British Medical Journal, 2, 37.

de Jong, R. N. (1940). Arch. Neurol. Psychiat., Chicago, 44, 1044.

Laurans, A. (1908). Paris Thesis, No. 210.

McIntyre, H. D. (1937). Ohio St. med. J., 33, 875

Petch, C. P. (1949). Lancet, 2, 405.

Shaby, J. A. (1949). Ibid., 2, 1153.

\section{Medical Memoranda}

\section{Sudden Simultaneous Arterial Embolism Involving All Four Limbs}

The following case is unusual enough to merit recording.

\section{CASE REPORT}

A man aged 58 was admitted to hospital complaining of difficulty in passing water. There was a history of a urethral discharge some 30 years previously, followed by a course of injections in the buttock, but no history of venereal sore. He had remained well until some 12 months before admission, when he noticed progressive difficulty in passing water. The stream got smaller and smaller and eventually stopped. At this point he attended a V.D. out-patients department, where a diagnosis of urethral stricture was made and his urinary retention was relieved after the passage of a curved sound. A blood W.R. taken at this time was found to be positive (strong). He did not attend for further treatment until retention occurred a second time some six months later. Instruments were again passed, after which he voided about $18 \mathrm{oz}$. (510 ml.) of urine loaded with pus. He was admitted to hospital the following day.

Examination revealed a rather pale ill-looking man. The presence of a stricture in the bulbous urethra was confirmed instrumentally; slight bleeding followed the passage of a medium-sized curved sound. Shortly afterwards, prior to a more detailed physical examination, he had a syncopal attack when paying a visit to the toilet. Loss of consciousness was brief: he was seen by a medical officer within a few minutes and ordered strict rest in bed, warmth, and copious fluids. During the afternoon his temperature rose to $102^{\circ} \mathrm{F}$. $\left(38.9^{\circ} \mathrm{C}\right.$.), but his general condition was described as satisfactory.

The next day he seemed to have recovered from his syncopal attack. There was hardly any urethral bleeding, and urine was passed quite satisfactorily. He was ordered to remain on strict rest in bed and to take copious fluids. His condition was satisfactory for the next five days, but the following night he complained to the nursing staff that his limbs had suddenly become painful, and on examination they were found to be discoloured.

Examination early the following morning showed a very ill-looking man with a dry brown tongue and labial herpes. Both hands and both forearms were cold and cyanosed and completely pulseless. Arterial pulsation could be felt in both antecubital fossae, but none at all below this level. Both legs were similarly cold and pulseless : the skin of the right leg was mottled blue and white; mottling was not present 
on the left side. Arterial pulsation could be felt above the inguinal ligament but not below this level ; the left femoral artery could be felt pulsating for a short distance below the inguinal ligament. It was impossible to detect pulsation in either popliteal fossae or at any point below this level. The skin of the scrotum was also deeply cyanosed. The patient was conscious and quite rational. The heart seemed to be beating strongly: a systolic murmur could be heard at the apex. There were rales at both lung bases. The colour and temperature of the skin of the trunk were normal.

A diagnosis of multiple arterial embolism was made: the prognosis was very poor if not hopeless, but death occurred sooner than was anticipated. The patient suddenly developed Cheyne-Stokes respiration and died within a matter of minutes a few hours after being examined and before any pathological examinations had been carried out.

\section{POST-MORTEM REPORT}

A post-mortem examination was made the following day, about twenty hours after death. The body was that of an elderly male. Sordes was present on the mouth. An infarct, which appeared to be of fairly recent origin, was found in the wall of the left ventricle: it did not involve the endocardial surface of the ventricle, which was of normal appearance, and no intracardiac thrombus was seen. Careful examination of the heart valves showed them to be normal. The coronary arteries and their main branches were laid open, but the expected thrombus was not found.

Striking changes were present in the ascending aorta and aortic arch. Projecting into the vessel lumen were several large exuberant thrombi attached to the base of atheromatous ulcers. The adventitia was thickened, and subsequent histological examination showed focal collections of lymphocytes and ruptured elastic fibres, suggesting the coexistence of both aortic syphilis and atheroma. No aneurysm was present.

A detailed dissection of the right femoral artery revealed an embolus with propagated thrombus extending to within 5 in. $(12.5 \mathrm{~cm}$.) of the inguinal ligament. There were signs of a diffuse infection in both lungs and a considerable amount of purulent material in the main bronchi.

The right kidney was scarred as a result of previous pyelonephritis; the left was normal apart from a recent infarct at the upper pole. The right ureter was dilated; the bladder showed cystitis with periureteric diverticula. A stricture was present in the bulbous urethra.

Cirrhosis of a coarse nodular type was present in the liver. The brain was oedematous, but no gross vascular lesion was found. Examination of the gastro-intestinal tract, thyroid, and adrenals revealed no lesion of any significance.

\section{Discussion}

Whereas the clinical picture left one in no doubt that emboli had lodged in all four main limb vessels, it was only at necropsy that the source of the emboli was revealed.

Aortic atheroma is a notoriously "silent" lesion, and in consequence is given far greater prominence in textbooks of pathology than in textbooks of medicine. Despite its frequency as a post-mortem finding, it is unusual for the swift arterial current to allow thrombi of any size to build up on the aortic wall even in advanced cases of atheromatous ulceration. Still less commonly do such thrombi become detached to block an artery farther down the vascular tree and cause clinical symptoms. Thus Kvale (1947), in an analysis of 100 cases of arterial occlusion studied at the Mayo Clinic, does not list it as an aetiological factor. It is possible, however, that cases do occur and masquerade under the diagnosis of arterial thrombosis. Learmonth (1948) describes cases in which an embolus has originated from a mural thrombus in a large artery.

In discussing the subject of multiple embolism a distinction must be drawn between multiple emboli occurring consecutively and multiple emboli occurring simultaneously. A succession of arterial emboli may punctuate the course of a number of diseased states. notably subacute infective endocarditis and auricular fibrillation.
On the other hand, multiple embclism occurring simultaneously has very rarely been recorded. Branch (1937) describes the case of a man aged 56 with a 10-years history of hypertension who developed simultaneous occlusion of both popliteal arteries. He lived for five days, during which time the occlusion spread proximally to both femoral arteries. Permission for a post-mortem examination was refused. Branch speculates on the cause, and observes that the literature mentions no previous case of simultaneous obstruction of the arteries of the lower extremities of sudden onset. Fiddian (1949) records the case of a man aged 69 who developed simultaneous embolism of both axillary arteries two and half months after a leg amputation. He does not suggest any source for the embolism, and no references are cited. I have been unable to trace any previously recorded cases of simultaneous occlusion of all four limb arteries.

My thanks are due to Dr. H. E. Vickers, consultant pathologist, Walton Hospital, for the post-mortem examination and for permission to summarize the post-mortem report.

C. D. Alergant, M.D., M.R.C.P., Consultant Venereologist, Newsham General Hospital.

\section{REFERENCES}

Branch, W. H. (1937). J. nat. med. Ass., 29, 89

Fiddian, J. V. (1949). British Medical Journal, 1, 480

Kvale, W. F. (1947). In Peripheral Vascular Diseases, by E. V. Allen. N. W. Barker, and E. A. Hines, jun. Saunders, Philadelphia and Learmonth, J. R. (1948). Edinb. med. J., 55, 449.

\section{Contamination of Streptomycin Solutions with Resistant Bacteria}

The recent coincidence of abscesses at the site of injection of streptomycin in three different patients under treatment for tuberculosis has drawn our attention to what is likely to be an increasing hazard of chemotherapy. Records of the hospital concerned showed that $10 \%$ of the patients given courses of streptomycin for tuberculosis in the previous five years had suffered from a similar abscess. Pus from the abscesses had been cultured in most instances, yielding growths of Staphylococcus aureus. The earlier cases of sepsis had appeared sporadically, so that their significance was overlooked, but it was now obvious that inquiry was necessary into the methods used for preparing and injecting the streptomycin.

Solutions of streptomycin were prepared the day before use by adding $2 \mathrm{ml}$. of sterile water to $1 \mathrm{~g}$. of the base (as the calcium chloride compound). Samples of such solutions were taken for culture by inoculating 5-ml. volumes of broth with two or three drops from syringes charged ready for injection. The bottles of broth were then posted to the laboratory. After incubation for five days, 6 out of 27 such samples yielded a growth of Staph. aureus. Swabs of equipment and materials yielded no pathogenic staphylococci. Two nurses shared the task of dissolving and injecting the streptomycin. One of them, Nurse A, proved to be a heavy nasal and skin carrier of Staph. aureus, and the other, Nurse B, a nasal carrier. The strains isolated from the three most recent abscesses, the streptomycin solutions, and the two nurses were typed with bacteriophage and their sensitivity to five antibiotics determined. All except one belonged to phage-type 53/77 and were resistant to streptomycin and penicillin but sensitive to aureomycin, oxytetracycline, and chloramphenicol. The exception was the strain isolated from Nurse B, which belonged to type 52A and was sensitive to all five antibiotics.

The survival of two of the streptomycin-resistant strains was studied in streptomycin solutions of 300.000 units per $\mathrm{ml}$. When $10^{2}$ viable cocci were added to $1 \mathrm{ml}$. of solution $25 \%$ survived after 24 hours at room temperature in the dark. With $10^{\mathrm{s}}$ cocci per $\mathrm{ml}$. approximately $1 \%$ survived in the same conditions. In contrast, similar tests on streptomycin-sensitive staphylococci with an inoculum of $10^{8}$ per $\mathrm{ml}$. showed that more than $99 \%$ were killed in 10 minutes, a finding consistent with the observations of 